\title{
SOME EXAMPLES IN DEGREE OF APPROXIMATION BY RATIONAL FUNCTIONS ${ }^{(1)}$
}

\author{
BY \\ D. AHARONOV AND J. L. WALSH
}

\begin{abstract}
We exhibit examples of (1) series that converge more rapidly than any geometric series where the function represented has a natural boundary, (2) the convergence of a series with maximum geometric degree of convergence yet having limit points of poles of the series everywhere dense on a circumference in the complement of $E$, (3) a Padé table for an entire function whose diagonal has poles everywhere dense in the plane and (4) a corresponding example for the table of rational functions of best approximation of prescribed type.
\end{abstract}

Specific examples are frequently of service in a developing theory, both to suggest new propositions and as counterexamples to refute possible conjectures. In the present paper we present some examples of this nature concerning degree of approximation by rational functions.

To be more explicit, we study $(\$ 1)$ examples of series that converge more rapidly than any geometric series where the function represented has a natural boundary, in $\$ 2$ the convergence of a series with maximum geometric degree of convergence yet having limit points of poles everywhere dense on a circumference in the complement of $E$. In $\$ 3$ we give an illustration of rational functions of degree $n$ in the Padé table for an entire function where the diagonal has poles everywhere dense in the plane, and in $\$ 4$ a corresponding example for the diagonal sequence in the analogous table of rational functions of best approximation.

1. Rapidly converging series. An infinite series

$$
f(z) \sim u_{1}(z)+u_{2}(z)+\cdots
$$

of rational functions of respective degrees $n$, is said to converge to $f(z)$ like $a$ geometric series on a set $E$ provided on that set for the sum $S_{n}(z)$ of the first $n$ terms of the second member of (1) we have

$$
\limsup _{n \rightarrow \infty}\left[\max \left|f(z)-S_{n}(z)\right|, z \text { on } E\right]^{1 / n}<1 .
$$

Received by the editors November 9, 1970.

AMS 1969 subject classifications. Primary 3070; Secondary 4140, 4141.

Key words and phrases. Approximation, rational functions, Padé table, best approximation, overconvergence.

( $\left.{ }^{1}\right)$ Research sponsored (in part) by USAFOSR Grant 69-1690.

Copyright (C) 1971, American Mathematical Society 
The series (1) is said to converge on E more rapidly than any (nontrivial) geometric series if we have

$$
\lim _{n \rightarrow \infty}\left[\max \left|f(z)-S_{n}(z)\right|, z \text { on } E\right]^{1 / n}=0 .
$$

If $u_{n}(z)$ is a rational function of degree $n$, if (2) is satisfied, and if there is no limit point of poles of the $u_{n}(z)$ on the closed region $E$, then the series (1) has important properties relative to convergence, notably that the series (1) overconverges, namely converges to $f(z)$ uniformly in a larger region containing $E$ in its interior. If under these same conditions (3) is satisfied, then [3] the series (1) converges, uniformly on compact sets in any region $E_{1}$ containing $E$ and containing no limit points of the poles of the $u_{n}(z)$. Moreover (3) is satisfied if $E$ is replaced by any closed subregion of $E_{1}$. If the function $f(z)$ is meromorphic at every finite point of the plane, and if the closed region $E$ is bounded, it is known [3] that there exists a sequence of rational functions of respective degrees $n$ whose poles lie in the poles of $f(z)$ and which satisfies (3). This raises the question as to whether (3) always implies that $f(z)$ has no natural boundary, a question which we proceed to answer here in the negative, by counterexamples.

THEOREM 1. Let $E$ be the closed unit disk $|z| \leqq 1$, and the points $\alpha_{1}, \alpha_{2}, \ldots$ chosen in the disk $E^{\prime}:|z|<3$ so that $\alpha_{1}$ lies on the circle $|z|=5 / 2$, the next two $\alpha_{k}$ are equally spaced on $|z|=8 / 3$, the next three $\alpha_{k}$ are equally spaced on $|z|=11 / 4$, and so on for the circles $|z|=3-1 / N$. We choose $A_{n}=1 / n^{n}$. Then the function

$$
f(z) \equiv \sum_{1}^{\infty} \frac{A_{k}}{z-\alpha_{k}}
$$

exists throughout $E^{\prime}$, is meromorphic there, is analytic on $E$ and satisfies

$$
\lim _{n \rightarrow \infty}\left[\max \left|f(z)-\sum_{k=1}^{n} \frac{A_{k}}{z-\alpha_{k}}\right|, z \text { on } E\right]^{1 / n}=0 .
$$

We note the relations

$$
\begin{gathered}
\sum_{k=n+1}^{\infty} A_{k}=\sum_{k=n+1}^{\infty} \frac{1}{k^{k}} \leqq \sum_{k=n+1}^{\infty} \frac{1}{(n+1)^{k}}=\frac{1}{n(n+1)^{n}}<A_{n}, \\
\lim _{n \rightarrow \infty}\left(\sum_{n+1}^{\infty} A_{k}\right)^{1 / n}=0 .
\end{gathered}
$$

If $z_{0}$ is a point in $E^{\prime}$ not an $\alpha_{n}$, we have for all $n\left|z_{0}-\alpha_{n}\right| \geqq 2 \delta(>0)$, where $\delta$ depends on $z_{0}$, and for $z$ in the neighborhood $\left|z-z_{0}\right|<\delta$ we have $\left|z-\alpha_{n}\right|>\delta$. Thus the series in (4) is dominated by the series $\sum A_{k} / \delta$, and by the Weierstrass $M$-test the series (4) converges uniformly in that neighborhood, so $f(z)$ is analytic in the neighborhood, in particular is analytic on $E$. The same proof shows that if $\alpha_{n}$ is arbitrary in $E^{\prime}$, then $f(z)-A_{n} /\left(z-\alpha_{n}\right)$ is analytic in a neighborhood of $\alpha_{n}$, so 
$f(z)$ is meromorphic in $E^{\prime}$. For $z$ on $E$ we have for every $n$ the inequality $\left|z-\alpha_{n}\right|>1$, whence

$$
f(z)-\sum_{k=1}^{n} \frac{A_{k}}{z-\alpha_{k}} \equiv \sum_{k=n+1}^{\infty} \frac{A_{k}}{z-\alpha_{k}}
$$

this last series is dominated by the first member of (6), and (7) implies (5). The rational function in (5) is of degree $n$, so (5) is of form (3). The analog of (5) holds for degree of approximation to $f(z)$ on $E$ by the rational functions of degrees $n$ of best approximation.

The function $f(z)$ clearly has the circle $|z|=3$ as a natural boundary.

A somewhat similar function but no longer with poles in $E^{\prime}$ is presented in

THEOREM 2. Let $E$ be the closed unit disk, $E^{\prime}$ the disk $|z|<3$, and the points $\alpha_{1}, \alpha_{2}, \ldots$ chosen all distinct but everywhere dense on the circle $|z|=3\left(\right.$ e.g. $\left.\alpha_{n}=3 e^{i n}\right)$. We choose $A_{n}=1 / n^{n}$. Then the function

$$
f(z) \equiv \sum_{1}^{\infty} \frac{A_{n} \alpha_{n}}{3\left(z-\alpha_{n}\right)}
$$

exists throughout $E^{\prime}:|z|<3$, is analytic there, and satisfies (where $\arg z=\arg \alpha_{n}$, $|z|<3)$

$$
\lim _{z \rightarrow \alpha_{n}}|f(z)|=\infty .
$$

Consequently, each $\alpha_{n}$ is a singularity of $f(z)$ and the circle $|z|=3$ is a natural boundary for $f(z)$.

It follows from the Weierstrass $M$-test that the series in (8), being dominated by the series $\sum A_{n} /(3-r)$ on the disk $|z| \leqq r<3$, is analytic on that disk and throughout $E^{\prime}$. We note too by (6) the inequality

$$
\sum_{k=N+1}^{\infty} A_{k}=A^{\prime}<A_{N} .
$$

Since the $\alpha_{n}$ are distinct, the function

$$
S_{N-1}(z)=\sum_{k=1}^{N-1} \frac{A_{k} \alpha_{k}}{3\left(z-\alpha_{k}\right)}
$$

is continuous at the point $z=\alpha_{N}$ and takes there some (finite) value $A$. Moreover, for $0<|z|<3, \arg z=\arg \alpha_{N}$, we have

$$
\left|z-\alpha_{N}\right|<\left|z-\alpha_{k}\right| \text { for } k>N \text { as } z \rightarrow \alpha_{N},
$$

whence

$$
f(z) \equiv S_{N-1}(z)+\frac{A_{N} \alpha_{N}}{3\left(z-\alpha_{N}\right)}+\sum_{k=N+1}^{\infty} \frac{A_{k} \alpha_{k}}{3\left(z-\alpha_{k}\right)}
$$


The first term in the second member approaches $A$, the second term is real and negative, of absolute value $A_{N} /\left|z-\alpha_{N}\right|$, and the third term is by (10) not greater in absolute value than

$$
\sum_{k=N+1}^{\infty} \frac{A_{k}}{\left|z-\alpha_{N}\right|}=\frac{A^{\prime}}{\left|z-\alpha_{N}\right|}<\frac{A_{N}}{\left|z-\alpha_{N}\right|}
$$

Consequently we have $\left(\arg z=\arg \alpha_{N}\right)$

$$
\lim _{z \rightarrow \alpha_{N}} \mathscr{R}[f(z)]=-\infty,
$$

so (9) is established, and $\alpha_{N}$ is a singularity of $f(z)$. Thus the circle $|z|=3$ is a natural boundary for $f(z)$ in $E^{\prime}$. We note incidentally that the proof of $(11)$ is valid too for $z \rightarrow \alpha_{N},|z|>3$, so the circle $|z|=3$ is also a natural boundary for $f(z)$ in $|z|>3$.

The function $f(z)$ in Theorem 2 is of significance for overconvergence, for we have for $z$ on $E$

$$
\left|f(z)-S_{n}(z)\right| \equiv \sum_{n+1}^{\infty}\left|\frac{A_{k} \alpha_{k}}{3\left(z-\alpha_{k}\right)}\right|<\sum_{n+1}^{\infty} \frac{A_{k}}{2},
$$

and by (7) we have (5). Of course (5) is of form (3). The analog of (5) holds for approximation to $f(z)$ on $E$ by the rational functions of degrees $n$ of best approximation.

A rational function $R_{m n}(z)$ of the form

$$
R_{m n}(z) \equiv \frac{a_{0} z^{m}+a_{1} z^{m-1}+\cdots+a_{m}}{b_{0} z^{n}+b_{1} z^{n-1}+\cdots+b_{n}}, \quad \sum\left|b_{i}\right| \neq 0,
$$

is said to be of type $(m, n)$. If the function $f(z)$ is continuous on a closed bounded set $E$, with no isolated points, for each type $(m, n)$ there exists a function of that type of best approximation to $f(z)$ on $E$; these functions can then be arranged [2] in a table, analogous to that of Padé,

$$
\begin{aligned}
& R_{00}, R_{10}, R_{20}, \ldots, \\
& R_{01}, R_{11}, R_{21}, \ldots, \\
& R_{02}, R_{12}, R_{22}, \ldots,
\end{aligned}
$$

Various sequences from this table have been studied [1], [2], [3]. The functions $R_{n n}(z)$ of the diagonal are precisely the rational functions of respective degrees $n$ of best approximation to $f(z)$ on $E$.

Perron has pointed out $[4$, p. 466] that there exists a function $f(z)$ such that the second row of the Padé table consists of rational functions whose poles are everywhere dense in the circle of convergence of $f(z)$. We prove now an analogous theorem concerning rational function of degree $n$ of best approximation to $f(z)$, the diagonal in the analog (12) of the Padé table. 


\section{Comparison series.}

THEOREM 3. Let $f(z)$ be a function regular in a closed bounded region $E$ and let $\left\{R_{n}(z)\right\}$ be the functions of degree $n$ of best approximation to $f(z)$ on E. Also assume

$$
\limsup _{n \rightarrow \infty}\left\|f(z)-R_{n}(z)\right\|^{1 / n}=A .
$$

(The norm is any of the ordinary norms.) Then a comparison series $T_{n}(z)$ may be found such that

(i) $\lim \sup _{n \rightarrow \infty}\left\|f(z)-T_{n}(z)\right\|^{1 / n}=A$,

(ii) the $T_{n}(z)$ are rational functions of degree $n$,

(iii) the set of poles of $\left\{T_{n}(z)\right\}$ form a dense subset in the complement $C(E)$.

Proof. Let $\left\{1 / \alpha_{0}^{(n)}\right\}_{n=1,2, \ldots}$ be a dense subset of $C(E)$ such that the $1 / \alpha_{0}^{(n)}$ are different from the poles of the $\left\{R_{n}(z)\right\}_{n=1,2, \ldots}$.

Let $A_{0}^{(n)}$ be so small that for $z \in E$ we have

$$
\lim _{n \rightarrow \infty}\left\|\frac{A_{0}^{(n)}}{1-\alpha_{0}^{(n)} z}\right\|^{1 /(n+1)}=0 .
$$

We define $T_{n+1}(z)=R_{n}(z)+A_{0}^{(n)} /\left(1-\alpha_{0}^{(n)} z\right)$, whence we have

$$
\begin{aligned}
\left\|f-R_{n+1}(z)\right\|^{1 /(n+1)} & \leqq\left\|f-T_{n+1}(z)\right\|^{1 /(n+1)} \\
& =\left\|f-R_{n}(z)-A_{0}^{(n)} /\left(1-\alpha_{0}^{(n)} z\right)\right\|^{1 /(n+1)} \\
& \leqq\left\|f-R_{n}\right\|^{1 /(n+1)}+\left\|A_{0}^{(n)} /\left(1-\alpha_{0}^{(n)} z\right)\right\|^{1 /(n+1)} .
\end{aligned}
$$

But

$$
\begin{gathered}
\limsup _{n \rightarrow \infty}\left\|f-R_{n+1}\right\|^{1 /(n+1)}=A, \quad \limsup _{n \rightarrow \infty}\left\|f-R_{n}\right\|^{1 /(n+1)}=A, \\
\lim _{n \rightarrow \infty}\left\|\frac{A_{0}^{(n)}}{1-\alpha_{0}^{(n)} z}\right\|^{1 /(n+1)}=0 .
\end{gathered}
$$

So we have

$$
\limsup _{n \rightarrow \infty}\left\|f-T_{n+1}(z)\right\|^{1 /(n+1)}=A,
$$

and the theorem follows.

We may replace the hypothesis (13) by

$$
\limsup _{n \rightarrow \infty}\left\|f(z)-R_{n}(z)\right\|^{1 / n} \leqq A,
$$

and deduce the conclusion

$$
\limsup _{n \rightarrow \infty}\left\|f(z)-T_{n}(z)\right\|^{1 / n} \leqq A .
$$

The rational functions $R_{n}(z)$ of best approximation are not necessarily unique, but we do not assert that the $T_{n}(z)$ are best approximating. The degree of convergence used in (13) and (14) does not distinguish between the two sequences, but presumably a more refined measure of degree of convergence would do so. 
In the study of equation (3), one might conjecture that (3) is possible only when the poles of the $S_{n}(z)$ lie in the poles of $f(z)$, or at least approach the singularities of $f(z)$. Theorem 3 of $\S 2$ effectively shows that the conjecture is false.

\section{Examples on Padé table.}

LEMMA $1[5$, p. 377]. Let $f(z)$ be a function analytic in a circle containing the origin with the expansion $f(z)=\sum_{k=0}^{\infty} a_{k} z^{k}$. Let $R_{n m}(z)$ be a rational function of type $(n, m)$ (i.e. a polynomial of order $n$ divided by a polynomial of order $m$ ) with the expansion $R_{n m}(z) \equiv \sum_{k=0}^{\infty} b_{k} z^{k}$ near the origin. Then if $a_{k}=b_{k}, k=0,1, \ldots, n+m$, $R_{n m}(z)$ is the Padé approximant of type $(n, m)$ to the function $f(z)$.

Proof. Denote $R_{n m}=P_{n} / Q_{n}$,

$$
f(z)-\frac{P_{n}(z)}{Q_{m}(z)}=\sum_{k=n+m+1}^{\infty} C_{k} z^{k} .
$$

If $\tilde{P}_{n}(z) / \widetilde{Q}_{m}(z)$ is a Padé approximant to $f(z)$, then by definition of the Padé approximant and (15) we get

$$
f(z)-\frac{\widetilde{P}_{n}(z)}{\widetilde{Q}_{m}(z)}=\sum_{k=n+m+1}^{\infty} d_{k} z^{k} .
$$

From (15) and (16) there follows

$$
\frac{P_{n}(z)}{Q_{m}(z)}-\frac{\widetilde{P}_{n}(z)}{\widetilde{Q}_{m}(z)}=\sum_{k=n+m+1}^{\infty} t_{k} z^{k} .
$$

So we have from (17)

$$
P_{n}(z) \widetilde{Q}_{m}(z)-\widetilde{P}_{n}(z) Q_{m}(z)=\sum_{k=n+m+1}^{\infty} V_{k} z^{k} .
$$

Since on the left side of (18) we have a polynomial of degree $n+m$ at most it follows that $V_{k}=0, k=n+m+1, \ldots$,

$$
P_{n}(z) / Q_{m}(z) \equiv \tilde{P}_{n}(z) / \tilde{Q}_{m}(z),
$$

which shows that $R_{n m}$ is the unique Padé approximant of type $(n, m)$.

EXAMPLE 1. Let

$$
f(z)=\frac{A_{0}}{1-\alpha_{0} z}+\frac{A_{1}}{1-\alpha_{1} z}+\sum_{v=0}^{\infty} h_{v} z^{v}=\sum_{k=0}^{\infty} t_{k} z^{k}
$$

such that $\sum_{v=0}^{\infty} h_{v} z^{v}$ is an entire function, $0<\left|\alpha_{0}\right|<\infty, 0<\left|\alpha_{1}\right|<\infty$. Let $g_{m 1}(z)$ $=b /(1-\beta z)+\sum_{v=0}^{m-1} C_{v} z^{v}=\sum_{k=0}^{\infty} d_{k} z^{k}$. In order that $g_{m 1}$ should be the Padé approximant of type $(m, 1)$ of $f(z)$ it is sufficient (by Lemma 1) that $d_{k}=t_{k}$, 
$k=0,1,2, \ldots, m+1$. So we have the set of equations

(20)

$$
\begin{aligned}
A_{0}+A_{1}+h_{0} & =b+C_{0}, \\
A_{0} \alpha_{0}+A_{1} \alpha_{1}+h_{1} & =b \beta+C_{1},
\end{aligned}
$$

$$
\begin{aligned}
\vdots & \vdots \\
A_{0} \alpha_{0}^{m-1}+A_{1} \alpha_{1}^{m-1}+h_{m-1} & =b \beta^{m-1}+C_{m-1} ; \\
A_{0} \alpha_{0}^{m}+A_{1} \alpha_{1}^{m}+h_{m} & =b \beta^{m}, \\
A_{0} \alpha_{0}^{m+1}+A_{1} \alpha_{1}^{m+1}+h_{m+1} & =b \beta^{m+1} .
\end{aligned}
$$

From the last two equations we get

$$
\beta=\left(A_{0} \alpha_{0}^{m+1}+A_{1} \alpha_{1}^{m+1}+h_{m+1}\right) /\left(A_{0} \alpha_{0}^{m}+A_{1} \alpha_{1}^{m}+h_{m}\right) .
$$

We now consider two cases:

$$
\left|\alpha_{0}\right|>\left|\alpha_{1}\right|, \quad\left|\alpha_{0}\right|=\left|\alpha_{1}\right| .
$$

Denote $\alpha_{1}=\tau \alpha_{0}$. Then

$$
\beta=\frac{\alpha_{0}^{m+1}}{\alpha_{0}^{m}} \frac{A_{0}+A_{1} \tau^{m+1}+h_{m+1} / \alpha_{0}^{m+1}}{A_{0}+A_{1} \tau^{m}+h_{m} / \alpha_{0}^{m}} .
$$

If $\left|\alpha_{1}\right|<\left|\alpha_{0}\right|$ then $|\tau|<1$. So $\beta \rightarrow \alpha_{0}$ as $m \rightarrow \infty$. If $\left|\alpha_{1}\right|=\left|\alpha_{0}\right|$ denote $\tau=e^{i \phi}$. Then

$$
\beta=\alpha_{0} \frac{A_{0}+\left(A_{1} \tau\right) \tau^{m}+h_{m+1} / \alpha_{0}^{m+1}}{A_{0}+A_{1} \tau^{m}+h_{m} / \alpha_{0}^{m}}
$$

For $\phi=2 \pi / l$ where $l$ is an integer we get for $\beta$ the $l$ limit points

$$
\alpha_{0} \frac{A_{0}+\left(A_{1} \tau\right) e^{2 \pi i k / l}}{A_{0}+A_{1} e^{2 \pi i k / l}}, \quad k=0,1, \ldots, l-1
$$

If $\phi / 2 \pi$ is an irrational number we get the whole circle (straight line)

$$
\alpha_{0} \frac{A_{0}+\left(A_{1} \tau\right) w}{A_{0}+A_{1} w}, \quad|w|=1,
$$

as limit points of $\beta$, as $m \rightarrow \infty$.

EXAMPle 2. Our aim is now to construct an example of an entire function $H(z)=\sum_{v=0}^{\infty} h_{v} z^{v}$ such that the diagonal in the Padé table will diverge in a dense subset of the plane. For convenience we consider the sequence $R_{n-1, n}(z)$ instead of the sequence $R_{n n}(z)$. The difference is only an additional additive constant.

Let $\alpha_{0}, 0<\left|\alpha_{0}\right|<\infty$, be arbitrary. Consider the function $A_{0} /\left(1-\alpha_{0} z\right)$, where $A_{0}$ is a constant that will be fixed later.

In order that $A_{0} /\left(1-\alpha_{0} z\right)$ will be $R_{01}(z)$ of $H(z)=\sum_{v=0}^{\infty} h_{v} z^{v}$, it is sufficient (Lemma 1) that

$$
A_{0}=h_{0}, \quad A_{0} \alpha_{0}=h_{1} .
$$


We now fix $A_{0}$ so that $\left|h_{0}\right|,\left|h_{1}\right|<1 / 1$ !. Next we consider the rational function

$$
\frac{b_{0}}{1-\beta_{0} z}+\frac{b_{1}}{1-\beta_{1} z}+\frac{b_{2}}{1-\beta_{2} z}
$$

where $\beta_{0}, 0<\left|\beta_{0}\right|<\infty$, is arbitrary, $\beta_{1}=q \widetilde{\beta}_{1}, \beta_{2}=q \widetilde{\beta}_{2}$, for $1>q>0$, and $q$ will be fixed later; $\widetilde{\beta}_{1} \neq \widetilde{\beta}_{2}$ are arbitrary (but $\widetilde{\beta}_{1}, \widetilde{\beta}_{2} \neq 0, \infty$ ). We now look for the conditions that this rational function will be $R_{23}(z)$ of $H(z)=\sum_{v=0}^{\infty} h_{v} z^{v}$ (only the coefficients $h_{0}, h_{1}$ are determined for the time being). Again, by Lemma 1, we have the set of equations:

$$
\begin{array}{cc}
b_{0}+b_{1}+b_{2} & =h_{0}, \\
b_{0} \beta_{0}+b_{1} \beta_{1}+b_{2} \beta_{2} & =h_{1}, \\
b_{0} \beta_{0}^{2}+b_{1} \beta_{1}^{2}+b_{2} \beta_{2}^{2} & =h_{2}, \\
\vdots \quad \vdots & \vdots \\
b_{0} \beta_{0}^{5}+b_{1} \beta_{1}^{5}+b_{2} \beta_{2}^{5} & =h_{5} .
\end{array}
$$

First $b_{0}$ is determined so that

$$
\left|b_{0} \beta_{0}^{j}\right|<1 / 2 \cdot 5 !, \quad j=2,3,4,5 .
$$

We next denote

$$
h_{0}-b_{0}=\lambda_{0}, \quad h_{1}-b_{0} \beta_{0}=\lambda_{1} .
$$

From (23) and (25) we get

$$
b_{1}+b_{2}=\lambda_{0}, \quad b_{1} \beta_{1}+b_{2} \beta_{2}=\lambda_{1} .
$$

Using the given conditions $\beta_{1}=q \widetilde{\beta}_{1}, \beta_{2}=q \widetilde{\beta}_{2}$ we have

$$
\left|\begin{array}{cc}
\lambda_{0} & 1 \\
\lambda_{1} & \tilde{\beta}_{2} q
\end{array}\right| /\left|\begin{array}{cc}
1 & 1 \\
\tilde{\beta}_{1} q & \tilde{\beta}_{2} q
\end{array}\right|=\frac{\lambda_{0} \tilde{\beta}_{2}-\lambda_{1} / q}{\tilde{\beta}_{2}-\tilde{\beta}_{1}} .
$$

We have a similar formula for $b_{2}$, so

$$
\left|b_{1}\right|,\left|b_{2}\right|<M / q, \quad M \text { depends only on } \lambda_{0}, \lambda_{1}, \widetilde{\beta_{1}}, \widetilde{\beta}_{2} \text {. }
$$

From (28) we get

$$
\begin{aligned}
\left|b_{1} \beta_{1}^{j}+b_{2} \beta_{2}^{j}\right| & =\left|b_{1} \widetilde{\beta}_{1}^{j} q^{j}+b_{2} \tilde{\beta}^{j} q^{j}\right| \\
& <2(M / q) q^{j} \operatorname{Max}\left(\left|\tilde{\beta}_{1}\right|^{j},\left|\tilde{\beta}_{2}\right|^{j}\right), \quad j=2,3,4,5 .
\end{aligned}
$$

Denote

$$
L=\underset{2 \leqq j \leqq 5}{\operatorname{Max}} \operatorname{Max}\left(\left|\tilde{\beta}_{1}\right|^{j},\left|\tilde{\beta}_{2}\right|^{j}\right) .
$$

From (29) and (30) we get

$$
\left|b_{1} \beta_{1}^{\prime}+b_{2} \beta_{2}^{\prime}\right|<2 M q L, \quad j=2,3,4,5 .
$$


If $q$ is sufficiently small we have

$$
\left|b_{1} \beta_{1}^{j}+b_{2} \beta_{2}^{j}\right|<1 / 2 \cdot 5 !, \quad j=2,3,4,5 .
$$

From (23), (24) and (32) we obtain

$$
\left|h_{j}\right|<1 / 5 !, \quad j=2,3,4,5 .
$$

Up to now the coefficients $h_{0}, h_{1}, \ldots, h_{5}$ have been determined; also we know that $R_{01}, R_{23}$ of the Padé table each has one arbitrary pole. In order to continue the procedure we have to construct $R_{67}$ (one arbitrary pole and six other poles because we have six coefficients of $H(z)$ already determined). So let $\sum_{k=0}^{6} C_{k} /\left(1-\gamma_{k} z\right)$ be a rational function such that $\gamma_{0}, 0<\left|\gamma_{0}\right|<\infty$, is arbitrary. $\gamma_{k}=q_{1} \tilde{\gamma}_{k}, 1>q_{1}>0$; $k=1,2, \ldots, 6 ; \tilde{\gamma}_{j} \neq \tilde{\gamma}_{l}, j \neq l, j, l \geqq 2$. In order that this function should be $R_{67}$ of $H(z)$ the following set of equations has to be fulfilled:

$$
\begin{aligned}
& C_{0}+C_{1}+\cdots+C_{6}=h_{0}, \\
& C_{0} \gamma_{0}+\cdots+C_{6} \gamma_{6}=h_{1} \text {, } \\
& C_{0} \gamma_{0}^{5}+\cdots+C_{6} \gamma_{6}^{5}=h_{5} \text {; } \\
& C_{0} \gamma_{0}^{6}+\cdots+C_{6} \gamma_{6}^{6}=h_{6} \text {, } \\
& C_{0} \gamma_{0}^{13}+\cdots+C_{6} \gamma_{6}^{13}=h_{13} \text {. }
\end{aligned}
$$

Exactly as we have done before we now choose $C_{0}$ so small that

$$
\left|C_{0} \gamma_{0}^{j}\right|<1 / 2 \cdot 13 !, \quad j=6, \ldots, 13 .
$$

Again, we define $\lambda_{j 1}=h_{j}-C_{0} \gamma_{0}^{j}, j=0, \ldots, 5$. From the first six equations in (33) we have

$$
\begin{array}{cc}
C_{1}+\cdots+C_{6}=\lambda_{01}, \\
C_{1} \gamma_{1}+\cdots+C_{6} \gamma_{6}=\lambda_{11}, \\
\vdots \quad \vdots \quad \vdots \\
C_{1} \gamma_{1}^{5}+\cdots+C_{6} \gamma_{6}^{5}= & \lambda_{51} .
\end{array}
$$

Using now the conditions $\gamma_{k}=q_{1} \tilde{\gamma}_{k}$ we get (in the same way as we obtained (28)),

$$
\left|C_{k}\right|<M_{1} / q_{1}, \quad k=1,2, \ldots, 6,
$$

$M_{1}$ depends only on $\left\{\lambda_{j 1}\right\} \cup\left\{\gamma_{l}\right\}, 0 \leqq j \leqq 5,1 \leqq l \leqq 6$. Exactly in the same manner as before we obtain

$$
\left|C_{1} \gamma_{1}^{s}+\cdots+C_{6} \gamma_{6}^{s}\right|<6 M_{1} L_{1} q_{1}, \quad j=6, \ldots,
$$

where $L_{1}$ is defined by

$$
L_{1}=\operatorname{Max}_{6 \leqq j \leqq 13} \operatorname{Max}_{1 \leqq k \leqq 6}\left(\left|\tilde{\gamma}_{k}\right|^{\jmath}\right) .
$$


For $q_{1}$ sufficiently small we get from (37)

$$
\left|C_{1} \gamma_{1}^{j}+C_{2} \gamma_{2}^{j}+C_{2} \gamma_{2}^{j}+\cdots+C_{6} \gamma_{6}^{j}\right|<1 / 2 \cdot 13 !, \quad j=6, \ldots, 13 .
$$

From (33), (34), and (39) we obtain

$$
\left|h_{j}\right|<1 / 13 !, \quad j=6, \ldots, 13 .
$$

In this way we continue to construct the subsequence of the diagonal of Padé table. Each of the terms of this subsequence has one arbitrary pole. It is clear that the $H(z)$ constructed is majorized by the exponential function and so is an entire function.

REMARK. By a slight change in the argument we could construct a subsequence of the diagonal such that each member of this subsequence would have more than one arbitrary pole. In fact the number of the arbitrary poles may increase monotonically to $\infty$. This is, of course, not needed here for the construction of the desired example.

\section{Examples on table (12).}

LemMa 1. Suppose $S_{p}(z)$ is a rational function of order $p$ having poles at $1 / \alpha_{j}$, $1 \leqq j \leqq p, 1 /\left|\alpha_{j}\right|>1,1 / \alpha_{j} \neq 1 / \alpha_{k}$ if $k \neq j,\left|1 / \alpha_{j}-1 / \alpha_{1}\right| \geqq 2 r>0$ for $2 \leqq j \leqq p$. Then there exists $\varepsilon=\varepsilon\left(\alpha_{1}, r, p\right)$ such that for any rational function $R_{p}(z)$ of order $p$ satisfying

$$
\sup _{|z| \leqq 1}\left|R_{p}(z)-S_{p}(z)\right|<\varepsilon\left|A_{1}\right|, \quad S_{p}(z)=\frac{A_{1}}{1-\alpha_{1} z}+\cdots+\frac{A_{p}}{1-\alpha_{p} z},
$$

$R_{p}(z)$ has at least one pole in the circle $\left|z-1 / \alpha_{1}\right| \leqq 2 r$.

Proof. Suppose the lemma is false. Then for a particular rational function $S_{p}(z)$ and $r>0$ there exist a sequence $\left\{\varepsilon_{n}\right\}$, such that $\varepsilon_{1} \geqq \varepsilon_{2} \geqq \cdots, \varepsilon_{n} \rightarrow 0$ and a sequence of rational functions $\left\{R_{p}^{(n)}(z)\right\}_{n=1}^{\infty}$ of order $p$ such that

$$
\sup _{|z| \leqq 1}\left|R_{p}^{(n)}(z)-S_{p}(z)\right|<\varepsilon_{n}\left|A_{1}\right|,
$$

and all the poles of $R_{p}^{(n)}(z)$ for any $n$ lie outside the disc $\left|z-1 / \alpha_{1}\right| \leqq 2 r$.

Denote the poles of $R_{p}^{(n)}(z)$ by $1 / \beta_{k n}, k=1,2, \ldots, p$. (It may occur that $1 / \beta_{k n}$ $=1 / \beta_{l n}$ for $l \neq k$.) Then by (4.2)

(4.3) $\sup _{|z|=1}\left|R_{p}^{(n)}(z)-S_{p}(z)\right|\left|\frac{\left(1-\alpha_{1} z\right) \cdots\left(1-\alpha_{p} z\right)\left(1-\beta_{1 n} z\right) \cdots\left(1-\beta_{p n} z\right)}{\left(z-\bar{\alpha}_{1}\right) \cdots\left(z-\bar{\alpha}_{p}\right)\left(z-\bar{\beta}_{1 n}\right) \cdots\left(z-\bar{\beta}_{p n}\right)}\right|<\varepsilon_{n}\left|A_{1}\right|$.

But the function on the left side of (4.3) is regular on $|z| \geqq 1$; so [1, §9.4]

$$
\sup _{|z| \geqq 1}\left|R_{p}^{(n)}(z)-S_{p}(z)\right|<\varepsilon_{n}\left|A_{1}\right| \frac{\left|\left(z-\bar{\alpha}_{1}\right) \cdots\left(z-\bar{\beta}_{p n}\right)\right|}{\left|\left(1-\alpha_{1} z\right) \cdots\left(1-\beta_{p n} z\right)\right|} \text {. }
$$

Now there is no loss of generality in assuming that $2 r<1 /\left|\alpha_{1}\right|-1$. So we have

$$
\sup _{\left|z-1 / \alpha_{1}\right|=r}\left|R_{p}^{(n)}(z)-S_{p}(z)\right|<\varepsilon_{n}\left|A_{1}\right| \frac{\left|\left(z-\bar{\alpha}_{1}\right) \cdots\left(z-\bar{\beta}_{p n}\right)\right|}{\left|\left(1-\alpha_{1} z\right) \cdots\left(1-\beta_{p n} z\right)\right|} \text {. }
$$


Let $R=1 /\left|\alpha_{1}\right|+r$. If $1 /\left|\alpha_{j}\right| \geqq 2 R$ we have for $\left|z-1 / \alpha_{1}\right|=r$

$$
1 /\left|1-\alpha_{j} z\right| \leqq 2 \text {. }
$$

Indeed, for $|z| \leqq R$ we have $\left|\alpha_{j}\right| \leqq 1 / 2 R$ so $\left|1-\alpha_{j} z\right| \geqq 1 / 2$. We have for $\left|z-1 / \alpha_{1}\right|=r$, $\left|1 / \alpha_{j}\right| \geqq 2 R$,

$$
\left|\left(z-\bar{\alpha}_{j}\right) /\left(1-\alpha_{j} z\right)\right| \leqq 2(R+1 / 2 R) .
$$

A similar result is obtained for $1 /\left|\beta_{j}\right| \geqq 2 R$.

Now assume that $1 /\left|\alpha_{j}\right|<2 R$. Then

$$
\left|\frac{z-\bar{\alpha}_{j}}{1-\alpha_{j} z}\right|=\left|\frac{\bar{\alpha}_{j}\left(1-z / \alpha_{j}\right)}{\alpha_{j}\left(z-1 / \alpha_{j}\right)}\right|<\frac{1+2 R^{2}}{\left|z-1 / \alpha_{j}\right|}, \quad 1 \leqq j \leqq p .
$$

But by our assumption $\left|1 / \alpha_{j}-1 / \alpha_{1}\right| \geqq 2 r$. So on the circle $\left|z-1 / \alpha_{1}\right|=r$ we have $\left|z-1 / \alpha_{j}\right| \geqq r$ for $2 \leqq j \leqq p$, and for $\left|z-1 / \alpha_{1}\right|=r_{1}$ we get $\left|1 / \alpha_{j}\right|<2 R$,

$$
\left|\left(z-\bar{\alpha}_{j}\right) /\left(1-\alpha_{j} z\right)\right|<\left(1+2 R^{2}\right) / r, \quad 1 \leqq j \leqq p .
$$

By our assumption on $R_{p}^{(n)}(z)$ we know that $\left|1 / \beta_{j n}-1 / \alpha_{1}\right| \geqq 2 r$ for $1 \leqq j \leqq p$. So we get a similar result for $1 /\left|\beta_{j n}\right|<2 R$. Combining (4.7) and (4.8) and the similar results for the $1 / \beta_{j}$ we get from (4.5)

$$
\sup _{\left|z-1 / \alpha_{1}\right|=r}\left|R_{p}^{(n)}(z)-S_{p}(z)\right|<\varepsilon_{n}\left|A_{1}\right|[2(R+1 / 2 R)]^{2 p}\left[\left(1+2 R^{2}\right) / r\right]^{2 p} .
$$

Now, the function $R_{p}^{(n)}(z)-S_{p}(z)$ has only the pole $1 / \alpha_{1}$ on the disc $\left|z-1 / \alpha_{1}\right| \leqq r$. So by integration of this function along the circle $\left|z-1 / \alpha_{1}\right|=r$ we have from (4.9)

$$
\left|A_{1}\right| /\left|\alpha_{1}\right|<\varepsilon_{n}\left|A_{1}\right|[2(R+1 / 2 R)]^{2 p}\left[\left(1+2 R^{2}\right) / r\right]^{2 p}=\varepsilon_{n} M(R, r, p)\left|A_{1}\right| .
$$

But now we let $n \rightarrow \infty$ and then $\varepsilon_{n} \rightarrow 0$ and we get the desired contradiction. (In fact we get the contradiction for $\varepsilon_{n}<1 / M(R, r, p)\left|\alpha_{1}\right|$. So $\varepsilon$ in the theorem can be taken as $\varepsilon=1 / 2 M(R, r, p)\left|\alpha_{1}\right|=\varepsilon\left(\alpha_{1}, r, p\right)$.)

Lemma 1, so far as concerns the existence of $\varepsilon=\varepsilon\left(\alpha_{1}, r, p\right)$ but not the specific formula (4.10), can be proved qualitatively by the properties of sequences of rational functions.

The existence of $R_{p}^{(n)}(z)$ satisfying (4.2) shows that the rational function $R_{p}^{(n)}(z)$ $-S_{p}(z)$ of degree $2 p$ approaches zero uniformly on $|z|<1$, hence $[1, \S 12.1]$ admits a subsequence which approaches zero in the extended plane with the omission of at most $2 p$ points. Since $S_{p}(z)$ has a pole in $1 / \alpha_{1}$ of fixed principal part independent of $n$, every such subsequence has at least one pole (necessarily belonging to $R_{p}^{(n)}(z)$ ) which approaches $1 / \alpha_{1}$; we see this by integrating $R_{p}^{(n)}(z)-S_{p}(z)$ over a circle $\left|z-1 / \alpha_{1}\right|=r_{1}<r$.

LemMA 2. Let $\eta>0, \varepsilon>0, r>0$, and $n+1$ complex numbers $C_{0}, C_{1}, \ldots, C_{n}$ be given. Also let $0<\left|\alpha_{1}\right|<1$. Then there exists $m>n$ and a rational function $S_{p}(z)$ of 
order $p=n+2$ with $a$ pole at $1 / \alpha_{1}$ and also at the points $\left\{1 / \alpha_{j}\right\}_{j=2}^{p}, \alpha_{j} \neq \alpha_{k}$ for $j \neq k$, $\left|1 / \alpha_{j}\right|>1$ such that for

$$
S_{p}(z)=\frac{A_{1}}{1-\alpha_{1} z}+\frac{A_{2}}{1-\alpha_{2} z}+\cdots+\frac{A_{p}}{1-\alpha_{p} z}=\sum_{k=0}^{\infty} \mu_{k} z^{k}
$$

we have

(a) $\mu_{k}=C_{k}, k=0,1, \ldots, n$,

(b) $\left|\mu_{n+1}\right|+\left|\mu_{n+2}\right|+\cdots<\eta$,

(c) $\left|\mu_{n+1}\right|,\left|\mu_{n+2}\right|, \ldots,\left|\mu_{m}\right|<1 / m !$,

(d) $\left|\mu_{m+1}\right|+\left|\mu_{m+2}\right|+\cdots<\varepsilon\left|A_{1}\right| / 4$,

(e) $\left|1 / \alpha_{j}-1 / \alpha_{1}\right| \geqq 2 r, 2 \leqq j \leqq p$.

Proof. Let $\left\{\alpha_{j}^{\prime}\right\}, 2 \leqq j \leqq p$, be arbitrary complex nonvanishing numbers, $\alpha_{j}^{\prime} \neq \alpha_{k}^{\prime}$ for $j \neq k$. Let $0<q<1$.

The exact value of $q$ will be determined later. Let $\alpha_{j}=q \alpha_{j}^{\prime}$ where $1 / \alpha_{j}$ are the poles of $S_{p}(z)$. We have by (a)

$$
\begin{array}{ccc}
A_{1}+A_{2}+\cdots+A_{p} & =\mu_{0}=C_{0}, \\
A_{1} \alpha_{1}+A_{2} \alpha_{2}+\cdots+A_{p} \alpha_{p} & =\mu_{1}=C_{1}, \\
\vdots & \vdots & \vdots \\
A_{1} \alpha_{1}^{n}+A_{2} \alpha_{2}^{n}+\cdots+A_{p} \alpha_{p}^{n} & =\mu_{n}=C_{n} .
\end{array}
$$

Also

$$
\begin{aligned}
A_{1} \alpha_{1}^{n+1}+A_{2} \alpha_{2}^{n+1}+\cdots+A_{p} \alpha_{p}^{n+1} & =\mu_{n+1} \\
A_{1} \alpha_{1}^{n+2}+\cdots+A_{p} \alpha_{p}^{n+2} & =\mu_{n+2}
\end{aligned}
$$

First we choose $m>n$ such that

$$
\left|\alpha_{1}\right|^{m+1}+\left|\alpha_{1}\right|^{m+2}+\cdots=\left|\alpha_{1}\right|^{m+1} /\left(1-\left|\alpha_{1}\right|\right)<\varepsilon / 8 .
$$

Now we determine $A_{1} \neq 0$ so that

$$
\begin{gathered}
\left|A_{1}\right|\left|\alpha_{1}\right|^{n+1}, \quad\left|A_{1}\right|\left|\alpha_{1}\right|^{n+2}, \ldots, \quad\left|A_{1}\right|\left|\alpha_{1}\right|^{m}<1 / 2 \cdot m !, \\
\left|A_{1}\right|\left|\alpha_{1}\right|^{n+1}+\left|A_{1}\right|\left|\alpha_{1}\right|^{n+2}+\cdots<\eta / 2 .
\end{gathered}
$$

From (4.12) we get

$$
\begin{array}{ccc}
A_{2}+\cdots+A_{p} & =C_{0}-A_{1}=C_{0}^{\prime}, \\
A_{2} \alpha_{2}+\cdots+A_{p} \alpha_{p} & =C_{1}-A_{1} \alpha_{1}= & C_{1}^{\prime}, \\
\vdots & \vdots & \vdots \\
A_{2} \alpha_{2}^{n}+\cdots+A_{p} \alpha_{p}^{n}= & C_{n}-A_{1} \alpha_{1}^{n}= & C_{n}^{\prime} .
\end{array}
$$


But $\alpha_{j}=q \alpha_{j}^{\prime}$ for $\alpha_{j}^{\prime} \neq \alpha_{l}^{\prime}$ if $j \neq l$. So $\alpha_{j} \neq \alpha_{l}$ for $j \neq l$. Also $p=n+2$. So we get from (4.17)

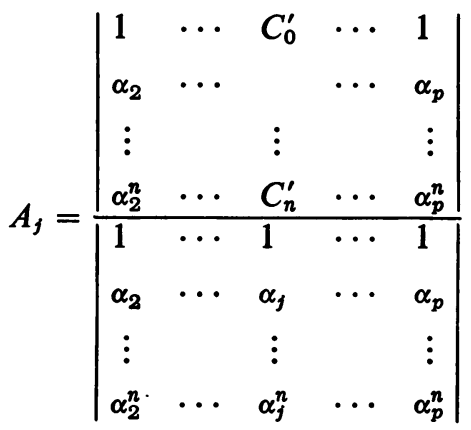

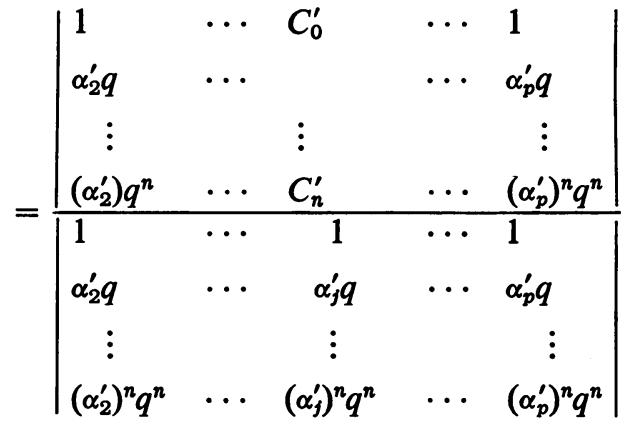

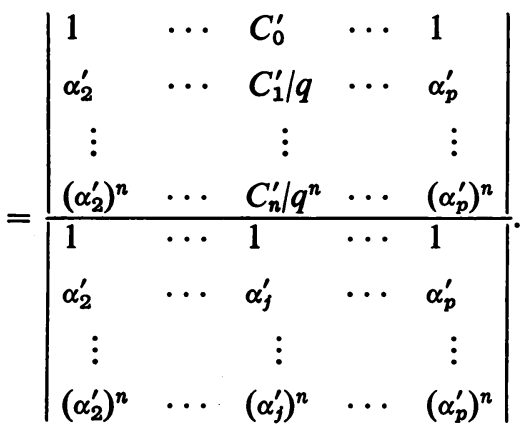

So we get (note that $0<q<1$ )

$$
\left|A_{j}\right|<M / q^{n}, \quad j=2,3, \ldots, p,
$$

where $M$ depends on $\left(C_{0}^{\prime}, \ldots, C_{n}^{\prime}\right)$ and $\left(\alpha_{2}^{\prime}, \ldots, \alpha_{p}^{\prime}\right)$ but not on $q$. Denote, now, $N=\max _{2 \leqq j \leqq p}\left|\alpha_{j}^{\prime}\right|$. Then from (4.18)

$$
\sum_{k=2}^{p}\left|A_{k}\right|\left|\alpha_{k}\right|^{n+j}<(p-1) M N^{n+j} q^{j}, \quad j=1,2, \ldots
$$

We now restrict $q$ to the interval $\left(0,1 / N\left(1+2 r\left|\alpha_{1}\right|\right)\right]$. Then

$$
\left|\alpha_{j}^{\prime} q\right|=\left|\alpha_{j}\right| \leqq N q \leqq \frac{\left|\alpha_{1}\right|}{1+2 r\left|\alpha_{1}\right|}, \quad 2 \leqq j \leqq p .
$$


So

$$
\left|\frac{1}{\alpha_{j}}-\frac{1}{\alpha_{1}}\right| \geqq\left|\frac{1}{\alpha_{j}}\right|-\left|\frac{1}{\alpha_{1}}\right| \geqq \frac{1+2 r\left|\alpha_{1}\right|}{\left|\alpha_{1}\right|}-\frac{1}{\left|\alpha_{1}\right|}=2 r
$$

for $2 \leqq j \leqq p$, and (e) is fulfilled.

We now restrict $q$ to a smaller interval, if needed, so that

$$
\frac{(p-1) M N^{n+1} q}{1-q N}<\min \left(\frac{1}{2 \cdot m !} \frac{\eta}{2}, \frac{\varepsilon}{8}\left|A_{1}\right|\right)
$$

From (4.19) we get, summing on $j$,

$$
\sum_{j=1}^{\infty} \sum_{k=2}^{p}\left|A_{k}\right|\left|\alpha_{k}\right|^{n+j}<\frac{(p-1) M N^{n+1} q}{1-q N}
$$

From (4.20) and (4.21) we clearly have

$$
\begin{aligned}
\sum_{j=1}^{\infty} \sum_{k=2}^{p}\left|A_{k}\right|\left|\alpha_{k}\right|^{n+j} & <\frac{\eta}{2} \\
\sum_{k=2}^{p}\left|A_{k}\right|\left|\alpha_{k}\right|^{n+j} & <\frac{1}{2 \cdot m !}, \quad j=1,2, \ldots, m-n, \\
\sum_{j=1}^{\infty} \sum_{k=2}^{p}\left|A_{k}\right|\left|\alpha_{k}\right|^{m+j} & <\frac{\varepsilon}{8}\left|A_{1}\right| .
\end{aligned}
$$

(b) follows from (4.13), (4.16) and (4.22), (c) follows from (4.13), (4.15) and (4.22), (d) follows from (4.13), (4.14) and (4.22), and the proof of Lemma 2 is complete.

LEMMA 3. Let $\left\{1 / \alpha_{1 k}\right\}_{1}^{\infty}$ be a sequence of points outside the unit disc, dense in $|z| \geqq 1$. Let $r_{1} \geqq r_{2} \geqq \cdots r_{n} \rightarrow 0$. Consider the sequence of discs $\left\{U_{k}:\left|z-1 / \alpha_{1 k}\right| \leqq 2 r_{k}\right\}$. Suppose $\left\{\tau_{k}\right\}_{1}^{\infty}$ is an arbitrary sequence of points such that $\tau_{k} \in U_{k}, k=1,2, \ldots$ Then $\left\{\tau_{k}\right\}_{1}^{\infty}$ form a dense subset of $|z| \geqq 1$.

Proof. Let $z_{0}$ be any point in $|z| \geqq 1$. Since $\left\{1 / \alpha_{k}\right\}$ forms a dense subset of $|z| \geqq 1$ we can construct a subsequence $\left\{1 / \alpha_{1 k_{l}}\right\}_{l=1}^{\infty}$ such that $1 / \alpha_{1 k_{l}} \rightarrow z_{0}$. Consider the sequence $\left\{\tau_{k_{l}}\right\}_{l=1}^{\infty}$. Then $\left|\tau_{k_{l}}-1 / \alpha_{1 k_{l}}\right| \leqq 2 r_{k_{l}} \rightarrow 0$ as $l \rightarrow \infty$. So $\left|\tau_{k_{l}}-z_{0}\right| \rightarrow 0$ as $l \rightarrow \infty$ and the proof is complete.

EXAMPLE. (Compare example in \$3.) We now construct an example of an entire function such that the diagonal in the table (12) for the unit disc and the sup norm diverges at a dense subset of $|z| \geqq 1$.

Let $\left\{1 / \alpha_{1 k}\right\}_{1}^{\infty}$ be a sequence of points outside the closed unit disc, dense in $|z|>1$. Let $r_{1} \geqq r_{2} \geqq \cdots$ such that $r_{n} \rightarrow 0$. Our aim is first to construct a sequence of rational functions $\left\{S_{p_{l}}(z)\right\}_{l=1}^{\infty}$ of orders $\left\{p_{l}\right\}_{l=1}^{\infty}$ respectively. These rational functions will have a simple pole at $\left\{1 / \alpha_{1 l}\right\}_{l=1}^{\infty}$ respectively. Then an entire function is constructed with the aid of these rational functions. The subsequence $\left\{R_{p_{l}}(z)\right\}_{l=1}^{\infty}$ in table (12) will have simple poles in the discs $\left|z-1 / \alpha_{1 l}\right| \leqq 2 r_{l}, l=1,2, \ldots$, and so our assertion will follow by Lemma 3. The construction of the $\left\{S_{p_{l}}(z)\right\}_{l=1}^{\infty}$ makes use of Lemma 2, 
while for the proof that the poles of $\left\{R_{p_{l}}(z)\right\}_{l=1}^{\infty}$ lie near poles of $\left\{S_{p_{l}}(z)\right\}_{l=1}^{\infty}$ we need Lemma 1.

So our first aim is to construct the sequence $\left\{S_{p_{l}}(z)\right\}_{l=1}^{\infty}$. For this aim we need also to construct sequences $\left\{m_{l}\right\}_{l=1}^{\infty},\left\{\eta_{l}\right\}_{l=2}^{\infty},\left\{\varepsilon_{l}\right\}_{l=1}^{\infty}$. Also we will use the given sequences $\left\{r_{l}\right\}_{l=1}^{\infty}$ and $\left\{1 / \alpha_{1 l}\right\}_{l=1}^{\infty}$ for the construction of $\left\{S_{p_{l}}(z)\right\}_{l=1}^{\infty}$.

We first define $S_{p_{1}}(z)$ and then successively the sequence $\left\{S_{p_{l}}(z)\right\}$. Let $p_{1}=1$; $S_{p_{1}}(z)=A_{11} /\left(1-\alpha_{11} z\right)=\sum_{k=0}^{\infty} \mu_{k}^{(1)} z^{k} ; A_{11}$ will be fixed later. Now $\varepsilon_{1}$ is chosen as $\varepsilon_{1}=\varepsilon\left(\alpha_{11}, r_{1}, p_{1}\right)$ where $\varepsilon\left(\alpha_{11}, r_{1}, p_{1}\right)$ is the function appearing in Lemma $1 ; m_{1}$ is defined as a solution of the inequality

$$
\left|\alpha_{11}\right|^{m_{1}+1} /\left(1-\left|\alpha_{11}\right|\right)<\varepsilon_{1} / 4
$$

$A_{11} \neq 0$ is now chosen small enough to give

$$
\left|A_{11}\right|\left|\alpha_{11}\right|^{k}=\left|\mu_{k}^{(1)}\right|<1 / m_{1} !, \quad k=0,1,2, \ldots, m_{1} .
$$

(In fact, since $\left|\alpha_{11}\right|<1$ it is sufficient to take $\left|A_{11}\right|<1 / m_{1}$ !.) Let $l \geqq 2$. Suppose now that $\left\{S_{p_{k}}(z)\right\}_{k=1}^{l-1},\left\{m_{k}\right\}_{k=1}^{l-1},\left\{\varepsilon_{k}\right\}_{k=1}^{l-1}$ have been defined. For reference we denote

$$
S_{p_{j}}(z)=\frac{A_{1 j}}{1-\alpha_{1 j} z}+\frac{A_{2 j}}{1-\alpha_{2 j} z}+\cdots+\frac{A_{p_{j} j}}{1-\alpha_{p_{j} j} z}=\sum_{k=0}^{\infty} \mu_{k}^{(j)} z^{k}
$$

We now use Lemma 2 for $n_{l}=m_{l-1}\left(n_{l}\right.$ instead of $n$ in Lemma 2), $p_{l}=n_{l+2}\left(p_{l}\right.$ instead of $p$ in Lemma 2), $\alpha_{1 l}$ (instead of $\alpha_{1}$ ), $r_{l}$ (instead of $r$ ),

$$
\eta_{l}=\min _{1 \leqq j \leqq l-1}\left|A_{1 j} \varepsilon_{j}\right| / 2^{l+2-j}
$$

( $\eta_{l}$ instead of $\eta$ ), $\mu_{k}^{(l)}$ (instead of $\mu_{k}$ ). The constants $C_{0}, C_{1}, \ldots, C_{n}$ appearing in Lemma 2 we take as the first $n$ (i.e., $n_{l}$ or $m_{l-1}$ in our notation) coefficients of the furiction $S_{p_{l-1}}$. Thus, $C_{k}=\mu_{k}^{(l-1)}, k=0,1, \ldots, m_{l-1}$. The $\varepsilon$ appearing in Lemma 2 will be taken as $\varepsilon_{l}=\varepsilon\left(\alpha_{1 l}, r_{l}, p_{l}\right)$ where $\varepsilon\left(\alpha_{1 l}, r_{l}, p_{l}\right)$ is the function appearing in Lemma 1.

After the new notation in Lemma 2 we use the result of Lemma 2 to get the function $S_{p_{l}}(z)$ and the integer $m_{l}(m$ in Lemma 2$)$ such that for

$$
S_{p_{l}}(z)=\frac{A_{1 l}}{1-\alpha_{1 l} z}+\cdots+\frac{A_{p_{l} l}}{1-\alpha_{p_{l} l} z}=\sum_{k=0}^{\infty} \mu_{k}^{(l)} z^{k}
$$

and for $m_{l}$ we have
(a) $\mu_{k}^{(l)}=\mu_{k}^{(l-1)}, \quad k=0,1, \ldots, m_{l-1}$,
(b) $\left|\mu_{m_{l-1}+1}^{(l)}\right|+\left|\mu_{m_{l-1}+2}^{(l)}\right|+\cdots<\eta_{l}=\min _{1 \leqq j \leqq l-1} \frac{\left|A_{1 j} \varepsilon_{j}\right|}{2^{l+2-j}}$
(c) $\left|\mu_{m_{l-1}+1}^{(l)}\right|,\left|\mu_{m_{l-1}+2}^{(l)}\right|, \ldots,\left|\mu_{m_{l}}^{(l)}\right|<1 / m_{l}$ !,
(d) $\left|\mu_{m_{l}+1}^{(l)}\right|+\left|\mu_{m_{l}+2}^{(l)}\right|+\cdots<\left(\varepsilon_{l} / 4\right)\left|A_{1 l}\right|$,
(e) $\left|1 / \alpha_{j l}-1 / \alpha_{1 l}\right| \geqq 2 r_{l}, \quad 2 \leqq j \leqq p_{l}$. 
In this way we have defined the sequences $\left\{S_{p_{l}}(z)\right\}_{l=1}^{\infty},\left\{m_{l}\right\}_{l=1}^{\infty}$. Notice the special definitions of $S_{p_{1}}(z), m_{1}$; the fact that $\eta_{1}$ was not defined, and that $(4.26(\mathrm{c}))$ is complementary to (4.24) (in (4.26) we have $l \geqq 2$ ). Also, it is worth noting that $S_{p_{l}}(z)$ agrees with the first $m_{l-1}+1$ coefficients of $S_{p_{l-1}}(z)$, the first $m_{l-2}+1$ coefficients of $S_{p_{l-2}}(z)$ and so on (as follows from (4.26(a)) after substituting $l-1$, $l-2$, instead of $l$ ).

We now are in a position to define our function $f(z)$. It will be convenient to set

$$
f(z)=\sum_{k=0}^{\infty} d_{k} z^{k}
$$

We now simply demand

$$
d_{k}=\mu_{k}^{(l)}, \quad k=0,1, \ldots, m_{l}, l=1,2, \ldots
$$

We recall that because of (4.26(a)) the function is well defined. It follows from (4.28) that, because of (4.26(c)) and (4.24), $f(z)$ is an entire function. We have (recall that the norm is the sup norm on $|z| \leqq 1)\left\|f(z)-S_{p_{1}}(z)\right\|=\| \sum_{k=m_{1}+1}^{\infty} d_{k} z^{k}$ $-\sum_{k=m_{1}+1}^{\infty} \mu_{k}^{(1)} z^{k} \|$; this is because of (4.28) for $l=1$. So

$$
\left\|f(z)-S_{p_{1}}(z)\right\| \leqq \sum_{k=m_{1}+1}^{\infty}\left|d_{k}\right|+\sum_{k=m_{1}+1}^{\infty}\left|\mu_{k}^{(1)}\right| .
$$

From (4.23) and the definition of $S_{p_{1}}(z)$ we have

$$
\sum_{k=m_{1}+1}^{\infty}\left|\mu_{k}^{(1)}\right|=A_{11} \frac{\left|\alpha_{11}\right|^{m_{1}+1}}{1-\left|\alpha_{11}\right|}<\frac{\varepsilon_{1}\left|A_{11}\right|}{4} .
$$

From (4.26(b)) we get, summing on $l$ and taking $j=1$,

$$
\begin{aligned}
\sum_{l=2}^{\infty} \sum_{k=1}^{\infty}\left|\mu_{m_{l-1}+k}^{(l)}\right| & <\sum_{l=2}^{\infty} \eta_{l} \\
& \leqq \frac{\left|\varepsilon_{1}\right|\left|A_{11}\right|}{2}\left(\frac{1}{4}+\frac{1}{8}+\cdots\right)=\frac{\left|\varepsilon_{1}\right|\left|A_{11}\right|}{4}
\end{aligned}
$$

We now deduce from (4.28)

$$
d_{k}=\mu_{k}^{(l)}, \quad m_{l-1}+1 \leqq k \leqq m_{l}, l=2,3, \ldots
$$

From (4.31) and (4.32) we clearly have

$$
\sum_{k=m_{1}+1}^{\infty}\left|d_{k}\right|<\frac{\left|\varepsilon_{1}\right|\left|A_{11}\right|}{4} .
$$

From (4.29), (4.30) and (4.33) we get

$$
\left\|f(z)-S_{p_{1}}(z)\right\|<\frac{\left|\varepsilon_{1}\right|\left|A_{11}\right|}{2} .
$$


We now obtain a similar result for $\left\|f(z)-S_{p_{l}}(z)\right\|, 2 \leqq l$. The calculation is very similar to the above calculation. Indeed, from (4.28) we have

$$
\left\|f(z)-S_{p_{l}}(z)\right\|<\sum_{k=m_{l}+1}^{\infty}\left|d_{k}\right|+\sum_{k=m_{l}+1}^{\infty}\left|\mu_{k}^{(l)}\right| .
$$

From (4.26(d)) we have

$$
\sum_{k=m_{l}+1}^{\infty}\left|\mu_{k}^{(l)}\right|<\frac{\varepsilon_{l}}{4}\left|A_{1 l}\right|
$$

Now a similar formula to (4.31) has to be obtained. For this objective, we change $l$ to $t$ in (4.26(b)) and sum on $t$ from $l+1$ to $\infty$, taking $j=l$. So we get

$$
\sum_{t=l+1}^{\infty} \sum_{k=1}^{\infty}\left|\mu_{m_{t-1}+k}^{(t)}\right|<\sum_{t=l+1}^{\infty} \eta_{t} \leqq \frac{\left|\varepsilon_{l}\right|\left|A_{1 l}\right|}{4}
$$

From (4.32) and (4.37) we get

$$
\sum_{k=m_{l}+1}^{\infty}\left|d_{k}\right|<\frac{\varepsilon_{l}\left|A_{1 l}\right|}{4}, \quad l=2,3, \ldots
$$

So from (4.35), (4.36) and (4.38) we deduce

$$
\left\|f(z)-S_{p_{l}}(z)\right\|<\varepsilon_{l}\left|A_{1 l}\right| / 2, \quad l=2,3, \ldots .
$$

We now come to the last step, namely, showing that the functions of best approximation have poles "near" $\left\{1 / \alpha_{1 l}\right\}$. Indeed, let $\left\{R_{p}(z)\right\}_{p=1}^{\infty}$ be the diagonal in the table (12). Consider the subsequence $\left\{R_{p_{l}}(z)\right\}$. Since these are functions of best approximation we have from (4.34) and (4.39)

$$
\left\|f(z)-R_{p_{l}}(z)\right\|<\varepsilon_{l}\left|A_{1 l}\right| / 2, \quad l=1,2, \ldots
$$

From (4.34), (4.39) and (4.40) we get

$$
\left\|S_{p_{l}}(z)-R_{p_{l}}(z)\right\|<\varepsilon_{l}\left|A_{1 l}\right|, \quad l=1,2, \ldots
$$

Recalling now (4.26(e)) and the way the $\left\{\varepsilon_{l}\right\}_{l=1}^{\infty}$ were chosen we deduce from Lemma 1 that $S_{p_{l}}(z)$ has a pole (at least one) in the disc $\left|z-1 / \alpha_{1 l}\right| \leqq 2 r_{l}$. So our assertion follows now from Lemma 3.

REMARK. There are possibilities for generalization. By minor changes one gets a similar generalization to that mentioned in the remark following the corresponding example in \$3. Also, one may consider other norms rather than the sup norms. For this aim we may use the fact that for a regular function $g(z)$ in $|z| \geqq 1$, the integral $\int_{0}^{2 \pi}\left|g\left(r e^{i \theta}\right)\right| d \theta, p>0$, is a monotonic decreasing function of $r$ in the interval $1 \leqq r \leqq \infty$. This enables us to change Lemma 1 and to get the above example for the $L_{p}$ norm. 


\section{REFERENCES}

1. J. L. Walsh, Interpolation and approximation by rational functions in the complex domain, Amer. Math. Soc. Colloq. Publ., vol. 20, Amer. Math. Soc., Providence, R. I., 1935.

2. - On approximation to an analytic function by rational functions of best approximation, Math. Z. 38 (1934), 163-176.

3. - On the overconvergence of certain sequences of rational functions of best approximation, Acta Math. 57 (1931), 411-435.

4. O. Perron, Die Lehre von den Kettenbrüchen, Chelsea, New York, 1929.

5. H. S. Wall, Analytic theory of continued fractions, Chelsea, New York, 1967.

UNIVERSITY OF MARYLAND,

College Park, Maryland 20742 